\title{
Urine afamin and afamin-creatinine ratio as biomarkers for kidney injury
}

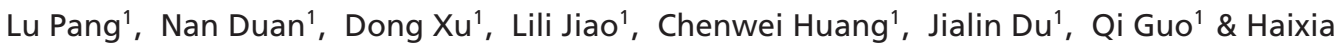 \\ $\mathrm{Li}^{*}, 1$ \\ ${ }^{1}$ Department of Clinical Laboratory, Peking University First Hospital, 100034, Beijing, PR China \\ *Author for correspondence: bdyylhx@126.com
}

\begin{abstract}
Aim: The aim of this study was to evaluate the urine afamin (UAFM) and afamin-creatinine ratio (AfCR) levels in patients with glomerulonephritis. Patients \& methods: We determined UAFM and AfCR of 247 healthy volunteers and 129 biopsy-proven glomerulonephritis patients. Results: Analytical evaluation study revealed the assay is a reliable and robust test for measuring uAFM. For reference intervals, UAFM and AfCR values were different significantly between males and females. UAFM and AfCR levels were significantly increased in patients with primary membranous nephropathy, IgA nephropathy and minimal change disease compared with healthy volunteers. UAFM and AfCR were positively correlated with urine albumin and albumin-creatinine ratio, respectively. Conclusion: Our study suggested that UAFM and AfCR may be attractive biomarkers for kidney injury.
\end{abstract}

First draft submitted: 21 April 2018; Accepted for publication: 31 August 2018; Published online: 15 November 2018

Keywords: afamin • ELISA • glomerulonephritis • kidney injury • urine

Afamin was discovered in 1994 by Lichtenstein et al. as the fourth member of the albumin superfamily, which comprises albumin, $\alpha$-fetoprotein and vitamin D-binding protein [1]. Araki et al. [2] determined the molecular weight of afamin as $75 \mathrm{kDa}$ with a carbohydrate content of $14 \%$. Afamin has been shown to be a specific binding protein for vitamin $\mathrm{E}$ via multiple binding sites, especially $\alpha$-tocopherol and $\gamma$-tocopherol, two of the most important forms of vitamin $\mathrm{E}[3,4]$.

Afamin is mainly expressed in the liver and secreted into the bloodstream but it can also be synthesized by brain capillary endothelial cells [5]. High concentrations of afamin have been described not only in plasma [4,6,7] but also in extravascular fluids such as urine [8-11], cerebrospinal fluid [4], peritoneal fluid [12], ovarian follicular and seminal fluids [4]. Although the presence of afamin in human urine has been verified by mass spectrometry [8-11] and western blot $[9,11]$, there is still a lack of a robust ELISA method for determination of urine afamin (uAFM).

Plasma afamin has been investigated in various types of carcinoma including ovarian cancer [13-16], gastric cancer [17,18], cholangiocarcinoma [19], colorectal cancer [20], uterine cervical cancer [21], breast cancer [22], papillary thyroid carcinoma [23,24] hepatocellular carcinoma [25], bladder cancer [8] - among others. In our recent proteomics study, we have discovered uAFM can serve as a biomarker candidate for primary membranous nephropathy (PMN) [26]. Meanwhile, uAFM has also been identified in several renal disorders, such as diabetic nephropathy [27], focal segmental glomerulosclerosis [9], IgA nephropathy (IgAN) [10,28] and pediatric idiopathic nephrotic syndrome [11]. Therefore, the concentration and clinical performance of uAFM need to be determined by appropriate methods in large study groups.

In the present study, we aimed to evaluate the uAFM and afamin-creatinine ratio (AfCR) levels in patients with glomerulonephritis and assess the analytical performance of an ELISA method for measurement of uAFM.

\section{Material \& methods}

\section{Samples collection}

In total, 2862 healthy volunteers for routine physical examinations were initially included. A detailed interview addressing personal history, family history, demographic information and laboratory examination was performed. The individuals with chronic inflammatory, metabolic diseases, hypertension, cancer, coronary heart disease, diabetes 
Table 1. Clinical information of 376 participants.

\begin{tabular}{|c|c|c|c|}
\hline Classifications & Males (n) & Females (n) & Age (years) ${ }^{\dagger}$ \\
\hline Healthy volunteers $(n=247)$ & $121(49.0 \%)$ & $126(51.0 \%)$ & $40.2 \pm 14.4$ \\
\hline PMN $(n=59)$ & $31(52.2 \%)$ & $28(47.5 \%)$ & $53.0 \pm 15.6$ \\
\hline $\operatorname{IgAN}(n=46)$ & $22(47.8 \%)$ & $24(52.2 \%)$ & $38.3 \pm 13.7$ \\
\hline$M C D(n=24)$ & $14(58.3 \%)$ & $10(41.7 \%)$ & $43.1 \pm 20.1$ \\
\hline
\end{tabular}

mellitus, renal disorders or abnormal laboratory examination were excluded. Finally, 247 healthy volunteers (121 males and 126 females) were selected as healthy control group in this study. 129 biopsy-proven glomerulonephritis patients from December 2015 to March 2017 were recruited in this study. Glomerulonephritis patients included PMN ( $=59)$, IgAN ( $=46)$ and minimal change disease (MCD, n = 24). The detail information of participants was shown in Table 1. Midstream morning urine samples of each participant were collected at Peking University First Hospital, Beijing, China. The collected samples were centrifuged at $2000 \times g$ for 10 min to remove cellular debris within $2 \mathrm{~h}$ and frozen in aliquots at $-80^{\circ} \mathrm{C}$ for further use. This study was approved by the ethics committee of Peking University First Hospital. Written informed consents were obtained from all participants.

\section{Biomarkers measurement}

uAFM was measured by ELISA (USCN Business Co., Ltd, Wuhan, China) in accordance with the manufacturer's recommendations. Urine creatinine (the Jaffe method) was measured by AU5800 automatic biochemical analyzer (Beckman Coulter, Inc., CA, USA). Urine albumin (uALB, the immunoturbidimetry method) was measured by IMMAGE 800 special protein analyzer (Beckman Coulter, Inc.). Albumin-creatinine ratio (ACR, mg/g) was calculated with urine creatinine and uALB. Serum creatinine (the Jaffe method) was measured by Synchron DXC800 automatic biochemical analyzer (Beckman Coulter, Inc.). Estimated glomerular filtration rate (eGFR) levels were calculated using the chronic kidney disease epidemiology collaboration equation recommended by the Kidney Disease Improving Global Outcomes [29].

\section{Analytical evaluation study}

Considering that the ELISA kit has been used for serum $[17,18]$ rather than urine, we evaluated precision, linearity and detection limit of the assay of this assay.

To verify the precision performance claims of the assay for uAFM, we performed a replication study according to the Clinical and Laboratory Standards Institute (CLSI) guideline EP15-A2 [30]. One pooled patient urine sample and one pooled healthy volunteer urine sample were aliquoted into $151.5 \mathrm{ml}$ plastic tubes for each concentration level and stored at $-80^{\circ} \mathrm{C}$ until analysis. We analyzed these samples one run per day with three replicate samples at each of two levels daily for 5 days (two levels $\times$ three replicates $\times 5$ days). We analyzed these samples one run per day with three replicate samples at each of two levels daily for 5 days (two levels $\times$ three replicates $\times 5$ days).

We validated the linearity of the ELISA method (claimed $0.78-50.00 \mathrm{ng} / \mathrm{ml}$ by manufacture) for uAFM according to the CLSI guideline EP6-A [31], using eight equally spaced concentrations. One pooled healthy volunteer urine sample and one pooled patient urine sample were used as a low- and a high-concentration pool. The low-concentration pool was mixed with the high-concentration pool in different proportions to create a series of concentrations spanning the measuring range: level 1, low only; level 2, 0.857 low +0.143 high; level 3, 0.714 low +0.286 high; level 4, 0.571 low +0.429 high; level 5, 0.429 low +0.571 high; level 6, 0.286 low +0.714 high; level 7, 0.143 low +0.857 high; level 8, high only. Each concentration was measured with three replicates randomly in a single run (eight levels $\times$ three replicates) and the default criteria were set at $12.2 \%$ (the larger of two coefficients of variation $[\mathrm{CVs}$ ] of repeatability precision in the Results section) for repeatability precision and $4.0 \mathrm{ng} / \mathrm{ml}$ for nonlinearity.

The limit of blank (LoB) and limit of detection (LoD) of the ELISA method were determined according to the CLSI guideline EP17-A [32]. LoB was determined by assaying the standard dilution buffer from the ELISA kit with 15 replicates one run per day for 4 days ( 15 replicates $\times 4$ days). To determine the LoD, seven low concentration samples ranged from LoB to $4 \times \mathrm{LoB}$ were measured two replicates one run per day for 6 days (seven samples $\times$ two replicates $\times 6$ days). 
Table 2. The repeatability and within-laboratory precisions.

\begin{tabular}{|c|c|c|c|c|}
\hline \multirow[t]{2}{*}{ Mean concentration $(\mathrm{ng} / \mathrm{ml})$} & \multicolumn{2}{|c|}{ Repeatability precision } & \multicolumn{2}{|c|}{ Within-laboratory precision } \\
\hline & $\mathrm{SD}(\mathrm{ng} / \mathrm{ml})$ & CV (\%) & $\mathrm{SD}(\mathrm{ng} / \mathrm{ml})$ & CV (\%) \\
\hline 38.9 & 4.8 & 12.3 & 4.8 & 12.3 \\
\hline 12.5 & 0.7 & 5.6 & 1.7 & 13.6 \\
\hline Manufacturer's claims & - & 10.0 & - & 12.0 \\
\hline
\end{tabular}

Figure 1. The first-order model of four equally spaced concentrations in validation of linearity. The solid line represents the linear model of four equally spaced concentration levels. Linear range of the method is 2.0-76.4 $\mathrm{ng} / \mathrm{ml}$. $\mathrm{n}=3$ per dilution level of the urine sample.

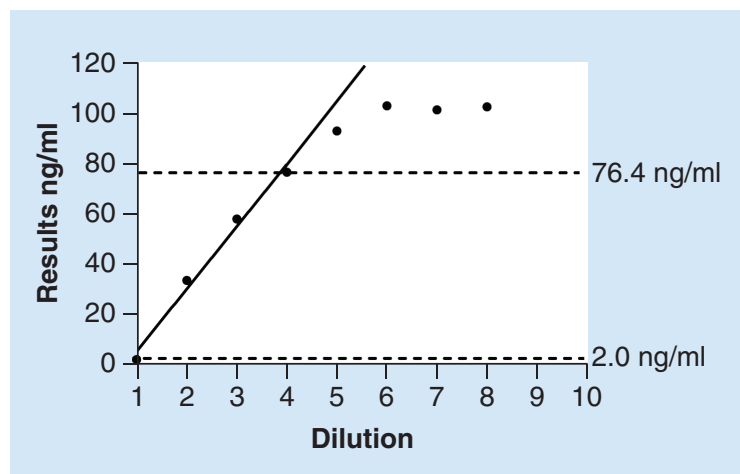

\section{Determination of reference intervals}

Reference intervals of uAFM were established according to the CLSI guideline C28-A3 [33]. Apart from uAFM $(\mathrm{ng} / \mathrm{ml})$, AfCR $(\mu \mathrm{g} / \mathrm{g})$ was also used to determine the reference intervals. Partitioning criteria are sex and age. Reference intervals were calculated by the nonparametric percentile method ( $95 \%$, one-tailed).

\section{Statistical analyses}

Data were statistically analyzed by the SPSS software version 19.0 for Windows (IBM, IL, USA). Graphs were prepared using GraphPad Prism version 6.0 (GraphPad Software, CA, USA). Values are represented as means \pm standard deviations for Gaussian distribution and medians (interquartile ranges) for non-Gaussian distribution. The nonlinear response pattern was checked with polynomial regression analysis in the verification of linearity. Comparisons of continuous variables were performed with the Mann-Whitney $U$ test or Kruskal-Wallis test, as appropriate. Relationships between continuous variables were tested with the Spearman coefficient of rank correlation. The ability of biomarkers in discriminating glomerulonephritis from healthy control was evaluated by the area under the receiver-operating characteristic curve (AUC-ROC) analysis with 95\% CI. A two-tailed p-value $<0.05$ was considered statistically significant.

\section{Results}

\section{Analytical evaluation study}

The CVs of repeatability and within-laboratory precision were $12.3 \%$ and $12.3 \%$ at a mean concentration of $38.9 \mathrm{ng} / \mathrm{ml}$, and $5.6 \%$ and $13.6 \%$ at a mean concentration of $12.5 \mathrm{ng} / \mathrm{ml}$ (Table 2 ). Although measured repeatability and within-laboratory precision were greater than the manufacturer's claims (Table 2), there were no statistically different values from the claims.

The low-concentration pool had a mean uAFM concentration of $2.0 \mathrm{ng} / \mathrm{ml}$ and the high-concentration pool of $102.4 \mathrm{ng} / \mathrm{ml}$. Deterioration in the linearity was observed when above the level 5 , so we narrowed the linear range to level 4. The default criteria for nonlinearity (Supplementary Figure 1) and repeatability precision were met. The first-order model of four equally spaced concentrations was shown in Figure 1. Therefore, the linear range of the method is $2.0-76.4 \mathrm{ng} / \mathrm{ml}$.

The LoB was $2.3 \mathrm{ng} / \mathrm{ml}$ calculated by the nonparametric percentile method (95\%, one-tailed) given a nonGaussian distribution of blank values. The LoD was $3.2 \mathrm{ng} / \mathrm{ml}$ calculated by 84 results according to the CLSI guideline EP17-A [32]. 

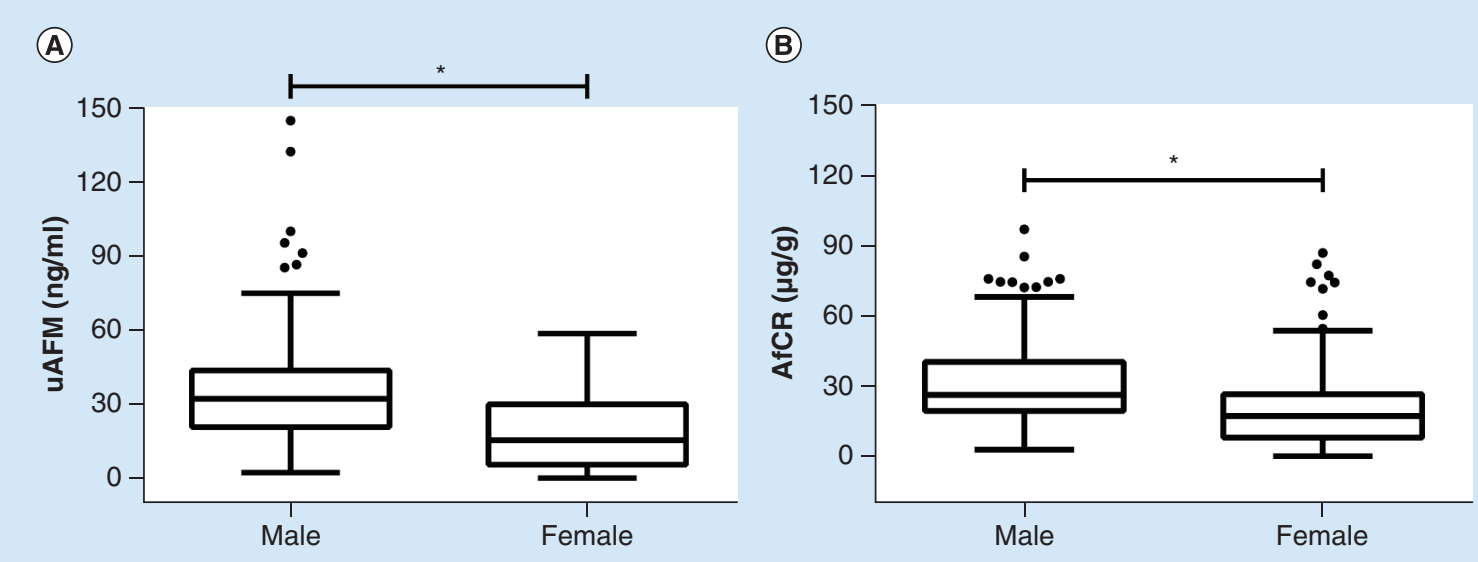

Figure 2. Distribution of urine afamin and afamin-creatinine ratio in healthy individuals according to sex groups. Box and whisker plots demonstrated gender-specific distributions of UAFM (A) and AfCR (B) in 121 males and 126 females. Mann-Whitney $U$ test was used to compare differences between sex groups. The detail information was shown in Supplementary Table 1.

${ }^{*} \mathrm{p}<0.05$.

AfCR: Afamin-creatinine ratio; uAFM: Urine afamin.

\begin{tabular}{|c|c|c|c|c|c|}
\hline \multirow[t]{2}{*}{ Sex groups } & \multirow[t]{2}{*}{$\mathrm{n}$} & \multicolumn{2}{|c|}{ uAFM (ng/ml) } & \multicolumn{2}{|c|}{$\operatorname{AfCR}(\mu \mathrm{g} / \mathrm{g})$} \\
\hline & & $r_{s}$ & p-value & $r_{s}$ & p-value \\
\hline Males & 121 & -0.137 & 0.134 & -0.047 & 0.612 \\
\hline Females & 126 & 0.036 & 0.687 & 0.169 & 0.058 \\
\hline
\end{tabular}

\section{Determination of reference intervals}

A histogram of uAFM in the healthy volunteers indicated a non-Gaussian distribution. Of the 247 healthy volunteers, 121 (49.0\%) were males and 126 (51.0\%) were females. Both uAFM and AfCR values were different significantly between male $s$ and females (Figure $2 \&$ Supplementary Table 1). For uAFM, the reference intervals were less than $86.3 \mathrm{ng} / \mathrm{ml}$ for males and less than $48.3 \mathrm{ng} / \mathrm{ml}$ for females. For AfCR, the reference intervals were less than $74.4 \mu \mathrm{g} / \mathrm{g}$ for males and less than $67.6 \mu \mathrm{g} / \mathrm{g}$ for females.

The mean age of the healthy volunteers was 40.2 . In sex subgroups, all individuals were categorized into five groups by age (20-29, 30-39, 40-49, 50-59 and $\geq 60$ years). No significant differences in uAFM and AfCR were observed across age groups (Supplementary Figure 2). Furthermore, Spearman coefficient of rank correlation analysis revealed no association between afamin-related variables (uAFM and AfCR) and age (Table 3).

\section{Clinical evaluation study}

The detail results of study participants in the clinical evaluation study were shown in Table 4. The distribution of uAFM and AfCR in healthy individuals and glomerulonephritis patients is displayed in Figure 3. The results indicated that uAFM and AfCR levels were significantly increased in patients with PMN, IgAN and MCD compared with healthy volunteers. Spearman coefficient of rank correlation analyses of all the 376 participants revealed significant positive correlations between uAFM and uALB $\left(r_{s}=0.620, p<0.001\right)$, and between AfCR and ACR $\left(r_{s}=0.545, p<0.001\right)$. The results of area under curve - receiver-operating characteristic (AUC-ROC) analysis evaluated the biomarkers in discriminating glomerulonephritis from healthy control are shown in Figure 4.

The mean eGFR of the healthy volunteers was $85.36 \pm 12.46 \mathrm{ml} / \mathrm{min} / 1.73 \mathrm{~m}^{2}$. There were no significant differences in afamin-related and albumin-related variables between group with an eGFR $<90 \mathrm{ml} / \mathrm{min} / 1.73 \mathrm{~m}^{2}$ and group with an eGFR $\geq 90 \mathrm{ml} / \mathrm{min} / 1.73 \mathrm{~m}^{2}$ in both males and females. (Supplementary Figure 3). Furthermore, 


\begin{tabular}{|c|c|c|c|c|c|c|}
\hline \multirow[t]{2}{*}{ Gender } & Study groups & $\mathbf{n}$ & uAFM (ng/ml) & $\operatorname{AfCR}(\mu \mathrm{g} / \mathrm{g})$ & uALB (mg/l) & ACR $(\mathrm{mg} / \mathrm{g})$ \\
\hline & Controls & 121 & $32.2(20.7-43.7)$ & $26.2(19.3-40.3)$ & $4.57(2.59-6.94)$ & $3.92(2.96-5.50)$ \\
\hline \multirow[t]{4}{*}{ Male: } & PMN & 31 & $37.3(28.9-52.7)^{\S}$ & $40.9(25.1-88.8)^{\S}$ & $2100.00(817.00-3260.00)^{\S}$ & $2553.06(837.78-4837.76)^{\S}$ \\
\hline & $\operatorname{IgAN}$ & 22 & $44.4(24.6-89.0)^{\S}$ & $71.0(33.2-142.9)^{\S}$ & $334.00(117.75-863.50)^{\S}$ & $381.78(222.41-798.76)^{\S}$ \\
\hline & MCD & 14 & $78.9(32.7-237.3)^{\S}$ & $155.0(70.6-304.3)^{\S}$ & $979.50(246.75-3837.50)^{\S}$ & $4199.21(481.03-5422.83)^{\S}$ \\
\hline & Controls & 126 & $15.4(5.5-9.9)$ & $17.2(8.0-26.6)$ & $3.90(2.19-6.22)$ & $4.63(3.54-6.29)$ \\
\hline \multirow[t]{3}{*}{ Female: } & PMN & 28 & $31.5(19.5-53.6)^{\S}$ & $83.3(42.4-145.2)^{\S}$ & $814.00(432.00-1975.00)^{\S}$ & $2556.69(1141.40-3367.80)^{\S}$ \\
\hline & $\operatorname{IgAN}$ & 24 & $28.3(15.5-52.5)^{\S}$ & $62.4(38.6-95.0)^{\S}$ & $318.00(77.60-630.25)^{\S}$ & $585.68(298.41-1052.97)^{\S}$ \\
\hline & $M C D$ & 10 & $27.5(18.9-32.0)^{\S}$ & $123.6(31.2-166.1)^{\S}$ & $216.50(45.70-3770.00)^{\S}$ & $1573.39(230.62-6638.48)^{\S}$ \\
\hline \multicolumn{7}{|c|}{$\begin{array}{l}\text { †Data were presented as medians (interquartile ranges). } \\
\ddagger \text { Mann-Whitney } U \text { test was used to compare differences between patients groups (PMN, IgAN or MCD) and controls. } \\
\S_{\mathrm{p}}<0.05 \text {. } \\
\text { ACR: Albumin-creatinine ratio; AfCR: Afamin-creatinine ratio; IgAN: IgA nephropathy; MCD: Minimal change disease; PMN: Primary membranous nephropathy; uAFM: Urine } \\
\text { afamin; UALB: Urine albumin. }\end{array}$} \\
\hline
\end{tabular}

(A)

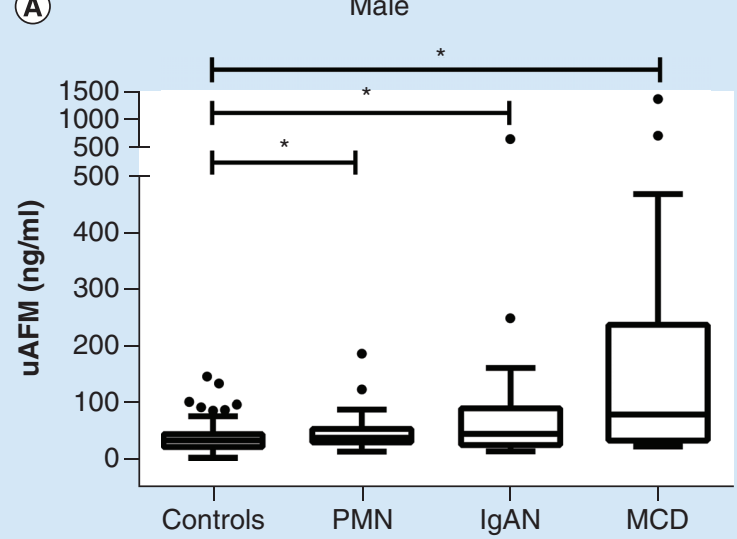

(C)

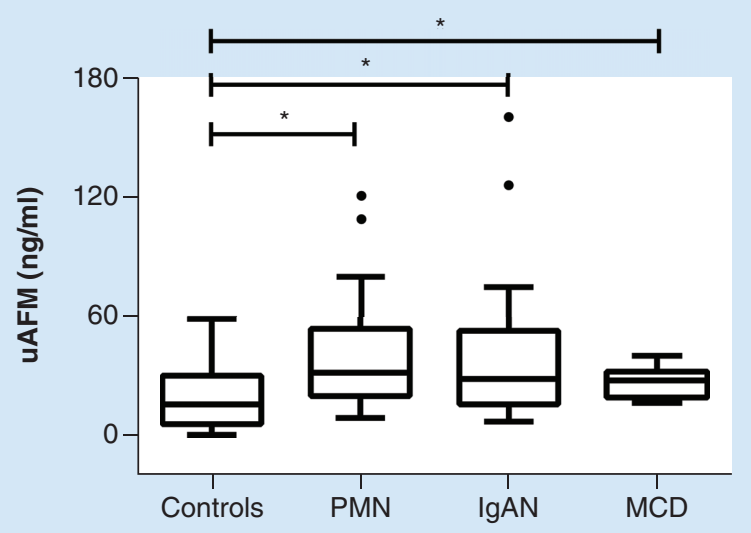

(B)

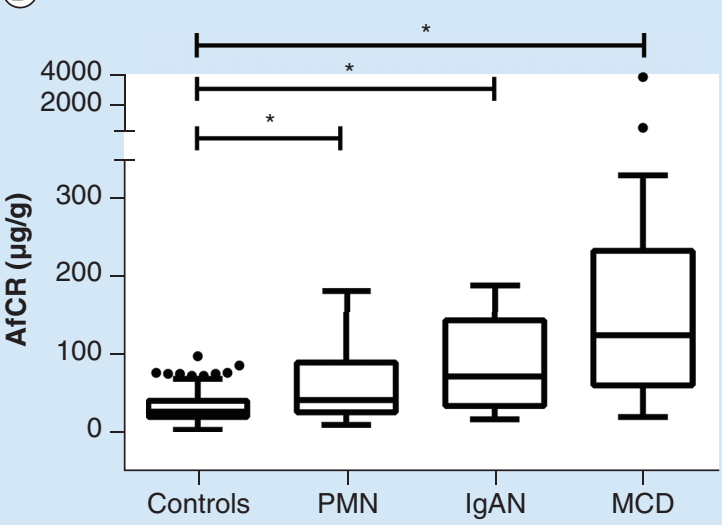

(D)

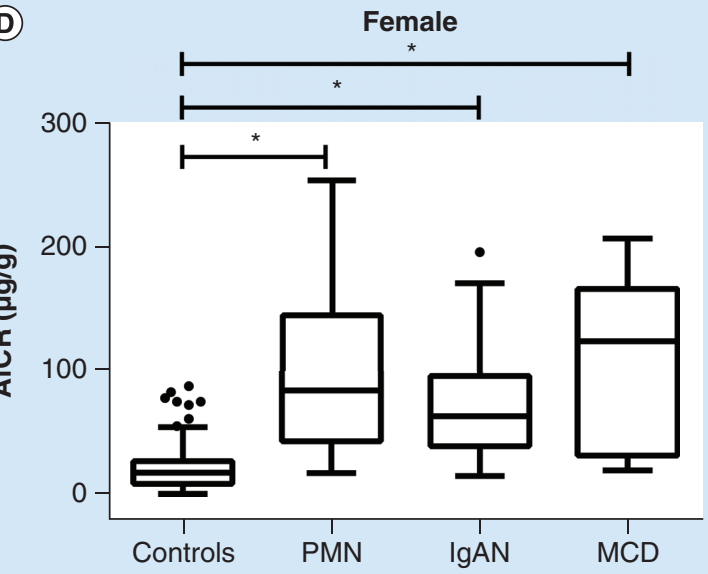

Figure 3. Distribution of urine afamin and afamin-creatinine ratio in healthy volunteers and patients. Box and whisker plots showed distribution of UAFM (A \& C) and AfCR (B \& D) in healthy volunteers (controls, $n=121$ for males and 126 for females) compared with patients with PMN ( $n=31$ for males and 28 for females), IgAN ( $n=22$ for males and 24 for females) and MCD ( $n=14$ for males and 10 for females). Mann-Whitney $\mathrm{U}$ test was used to compare differences.

$* p<0.05$.

ACR: Albumin-creatinine ratio; AfCR: Afamin-creatinine ratio; IgAN: IgA nephropathy; MCD: Minimal change disease; PMN: Primary membranous nephropathy; uAFM: Urine afamin. 


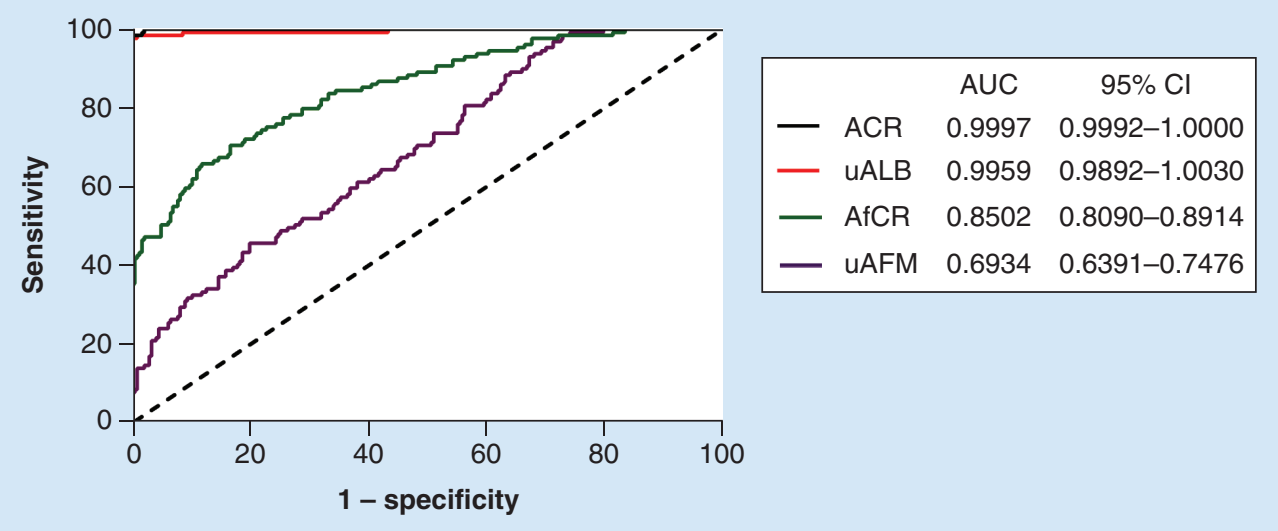

Figure 4. Area under the receiver-operating characteristic curve analysis of urine afamin, afamin-creatinine ratio, urine albumin and albumin-creatinine ratio in discriminating glomerulonephritis from healthy control. For color figures see online at: https://www.futuremedicine.com/doi/10.2217/bmm-2017-0126 ACR: Albumin-creatinine ratio; AfCR: Afamin-creatinine ratio; AUC: Area under the curve; uAFM: Urine afamin; UALB: Urine albumin.

Spearman coefficient of rank correlation analysis revealed no association between afamin-related variables (uAFM and AfCR) and eGFR, and between albumin-related variables (uALB and ACR) and eGFR.

\section{Discussion}

We aimed to evaluate the uAFM and AfCR levels in patients with glomerulonephritis. Our study provided support that the assay is a reliable and robust test for measuring uAFM. In the clinical evaluation study, we found that uAFM and AfCR were significantly increased in patients with PMN, IgAN and MCD. Furthermore, uAFM and AfCR were positively correlated with uALB and ACR, respectively, rather than eGFR. Our results indicate that uAFM and AfCR are biomarkers for glomerular barrier function, rather than glomerular filtration function.

Urinary proteins are mainly composed of plasma proteins filtered by the glomerular filtration membrane and proteins secreted from the urinary tract, especially the renal tubular epithelial cell [34]. Afamin is mainly expressed in the liver and secreted into the bloodstream [35]. Apart from liver and brain capillary endothelial cells [5], it has been shown that afamin mRNA can also expressed in kidney (Unigene Hs.168718). uAFM is mainly filtered by the glomerular filtration membrane. Whether the UAFM is partly secreted by the renal tubular epithelial cell still needs further research. Our results showed that uAFM and AfCR were positively correlated with uALB and ACR, respectively, but not with eGFR. These results indicated that uAFM and AfCR are biomarkers for glomerular barrier function, but not glomerular filtration function. Along with the question whether the renal tubular epithelial cell can synthesize afamin, another question whether the uALB can reflect the renal tubular function needs to be resolved. In our study, although the ability of AfCR to predict kidney injury is poor with an AUC-ROC (0.8502) compared with the ACR with an AUC-ROC (0.9997), our preliminary study showed uAFM and AfCR are potential biomarkers for glomerular barrier function. Whether the newfound biomarkers can predict early kidney injury and the performance when combined with other renal biomarkers need further study.

Several proteomics studies have discovered uAFM can serve as a biomarker candidate in multiple renal diseases. Rao et al. [27] identified uAFM as the urinary biomarker for diabetic nephropathy. Sedic et al. [11] detected the expression of uAFM from pediatric idiopathic nephrotic syndrome patients for the first time by combined proteomics and metabolomics approach based on label-free mass spectrometry. In a focal segmental glomerulosclerosis rat model, Zhao et al. [9] found uAFM was gradually increased during nephropathy progression by proteomics approach, indicating that uAFM may be associated with disease severity. In IgAN, Mucha et al. [28] found increased uAFM urine proteomics, and Kalantari et al. [10] suggested uAFM is predictive biomarker for severity of IgAN by label-free proteomics approach. Vivekanandan-Giri et al. [36] reported altered uAFM in chronic kidney disease by urinary glycoprotein profile proteomics, indicating the important role of glycoprotein as an early marker for kidney injury. However, all of the studies are mass spectrometry-based quantitative proteomics. Although it has become a powerful technology for the discovery of new biomarkers [37], hundreds of candidate biomarker proteins are often 
listed, awaiting validation [8]. It is the first time to validate the concentration of uAFM by the ELISA method in our study.

For quantification of uAFM, Chen et al. [8] performed a multiple reaction monitoring-mass spectrometry (MRM-MS) assay to determine the concentration of uAFM, which was the first time of quantification of uAFM. However, the MRM-MS assay is relatively quantificative and costly. Moreover, it needs specialized laboratory and professional technology. The knowledge of the absolute concentration of uAFM in healthy volunteers and diseased patients is still limited. ELISA is currently the most common method for measuring the concentration of a target protein in biological samples, with advantages including low cost and widespread application [38]. It is the first time to determine the absolute concentration of uAFM by ELISA, which will make the clinical routine application of uAFM possible.

Apart from the activity of binding vitamin E, it is shown that afamin plays an important role in cellular antioxidative defence [11]. For example, Heiser $e t$ al. [39] showed that the addition of afamin to cortical chicken neurons exposed to oxidative stress can protect cortical neurons from cell death. Oxidative stress plays a major role in pathogenesis and progression of renal injury [40], especially in diabetic nephropathy [41]. However, whether the afamin has the function of kidney protection needs to be further investigated.

This study has several limitations. On the one hand, we did not perform the all-sided methodology evaluation. The analyte stability and biological variability need to be evaluated in the future studies. On the other hand, it still requires evaluation of clinical samples from a larger and equally sample sizes to verify our results.

\section{Conclusion}

Our study provided support that our ELISA method is a reliable and robust test for measuring uAFM. uAFM and AfCR may be attractive biomarkers for kidney injury.

\section{Summary points}

- It has been reported that high concentrations of afamin have been described not only in plasma but also in extravascular fluids such as urine, cerebrospinal fluid and peritoneal fluid.

- In our recent proteomics study, we have discovered urine afamin (UAFM) can serve as a biomarker candidate for primary membranous nephropathy.

- Our study provided support that our ELISA method is a reliable and robust test for measuring UAFM.

- In order to avoid interference from concentration and dilution of urine, we develop a new biomarker, afamin-creatinine ratio (AfCR).

- For reference intervals study, both UAFM and AfCR values were different significantly between males and females.

- UAFM and AfCR levels were significantly increased in patients with primary membranous nephropathy, IgA nephropathy and minimal change disease compared with healthy volunteers.

- Significant positive correlations were observed between UAFM and UALB, and between AfCR and ACR.

- There were no significant differences in afamin-related and albumin-related variables between group with an estimated glomerular filtration rate $<90 \mathrm{ml} / \mathrm{min} / 1.73 \mathrm{~m}^{2}$ and group with an estimated glomerular filtration rate $\geq 90 \mathrm{ml} / \mathrm{min} / 1.73 \mathrm{~m}^{2}$ in both males and females.

- Our results indicate that UAFM and AfCR are biomarkers for glomerular barrier function, rather than glomerular filtration function.

\section{Supplementary data}

To view the supplementary data that accompany this paper please visit the journal website at: www.futuremedicine.com/doi/full/10.2217/bmm-2018-0126

Financial \& competing interests disclosure

The authors have no relevant affiliations or financial involvement with any organization or entity with a financial interest in or financial conflict with the subject matter or materials discussed in the manuscript. This includes employment, consultancies, honoraria, stock ownership or options, expert testimony, grants or patents received or pending, or royalties.

No writing assistance was utilized in the production of this manuscript. 
Ethical conduct of research

The authors state that they have obtained appropriate institutional review board approval or have followed the principles outlined in the Declaration of Helsinki for all human or animal experimental investigations. In addition, for investigations involving human subjects, informed consent has been obtained from the participants involved.

\section{Author's contributions}

L Pang and H Li contributed to the conception or design of the work. L Pang, N Duan, D Xu and Q Guo contributed to the acquisition of data for the work. L Pang, L Jia and C Huang contributed to the analysis of data for the work. L Pang, J Du and H $\mathrm{Li}$ contributed to the interpretation of data for the work. L Pang, N Duan, D Xu and $\mathrm{H}$ Li contributed to the drafting the work. $\mathrm{L}$ Jiao, C Huang, J Du and Q Guo contributed to the revising critically for important intellectual content. All the authors contributed to the final approval of the version to be published and agreement to be accountable for all aspects of the work in ensuring that questions related to the accuracy or integrity of any part of the work are appropriately investigated and resolved.

\section{Open access}

This work is licensed under the Attribution-NonCommercial-NoDerivatives 4.0 Unported License. To view a copy of this license, visit http://creativecommons.org/licenses/by-nc-nd/4.0/

\section{References}

Papers of special note have been highlighted as: • of interest; $\bullet \bullet$ of considerable interest

1. Lichenstein HS, Lyons DE, Wurfel MM et al. Afamin is a new member of the albumin, alpha-fetoprotein, and vitamin D-binding protein gene family. J. Biol. Chem. 269(27), 18149-18154 (1994).

- Discovered afamin as the fourth member of the albumin superfamily.

2. Araki T, Haupt H, Hermentin P et al. Preparation and partial structural characterization of alpha1T-glycoprotein from normal human plasma. Arch. Biochem. Biophys. 351(2), 250-256 (1998).

3. Voegele AF, Jerkovic L, Wellenzohn B et al. Characterization of the vitamin E-binding properties of human plasma afamin. Biochemistry 41(49), 14532-14538 (2002).

4. Jerkovic L, Voegele AF, Chwatal S et al. Afamin is a novel human vitamin E-binding glycoprotein characterization and in vitro expression. J. Proteome Res. 4(3), 889-899 (2005).

- Describes the purification and characterization of a novel vitamin E-binding glycoprotein, afamin, from human plasma.

5. Kratzer I, Bernhart E, Wintersperger A et al. Afamin is synthesized by cerebrovascular endothelial cells and mediates alpha-tocopherol transport across an in vitro model of the blood-brain barrier. J. Neurochem. 108(3), 707-718 (2009).

6. Dieplinger B, Egger M, Gabriel C et al. Analytical characterization and clinical evaluation of an enzyme-linked immunosorbent assay for measurement of afamin in human plasma. Clin. Chim. Acta 425, 236-241 (2013).

-• Quantitative measurement of afamin in human plasma.

7. Kronenberg F, Kollerits B, Kiechl S et al. Plasma concentrations of afamin are associated with the prevalence and development of metabolic syndrome. Circ. Cardiovasc. Genet. 7(6), 822-829 (2014).

8. Chen YT, Chen HW, Domanski D et al. Multiplexed quantification of 63 proteins in human urine by multiple reaction monitoring-based mass spectrometry for discovery of potential bladder cancer biomarkers. J. Proteomics 75(12), 3529-3545 (2012).

-• First quantification of urine afamin (uAFM) by multiple reaction monitoring-mass spectrometry.

9. Zhao M, Li M, Li X, Shao C, Yin J, Gao Y. Dynamic changes of urinary proteins in a focal segmental glomerulosclerosis rat model. Proteome Sci. 12, 42 (2014).

-• Found UAFM was gradually increased during focal segmental glomerulosclerosis progression by proteomics approach, indicating that uAFM may be associated with disease severity.

10. Kalantari $S$, Rutishauser D, Samavat $S$ et al. Urinary prognostic biomarkers and classification of IgA nephropathy by high resolution mass spectrometry coupled with liquid chromatography. PLoS ONE 8(12), e80830 (2013).

11. Sedic M, Gethings LA, Vissers JP et al. Label-free mass spectrometric profiling of urinary proteins and metabolites from paediatric idiopathic nephrotic syndrome. Biochem. Biophys. Res. Commun. 452(1), 21-26 (2014).

12. Seeber BE, Czech T, Buchner $\mathrm{H}$ et al. The vitamin E-binding protein afamin is altered significantly in the peritoneal fluid of women with endometriosis. Fertil. Steril. 94(7), 2923-2926 (2010).

13. Jackson D, Craven RA, Hutson RC et al. Proteomic profiling identifies afamin as a potential biomarker for ovarian cancer. Clin. Cancer Res. 13(24), 7370-7379 (2007).

14. Dieplinger H, Ankerst DP, Burges A et al. Afamin and apolipoprotein A-IV: novel protein markers for ovarian cancer. Cancer Epidemiol. Biomarkers Prev. 18(4), 1127-1133 (2009). 
15. Aktas B, Kasimir-Bauer S, Wimberger P, Kimmig R, Heubner M. Utility of mesothelin, L1CAM and Afamin as biomarkers in primary ovarian cancer. Anticancer Res. 33(1), 329-336 (2013).

16. Melmer A, Fineder L, Lamina C et al. Plasma concentrations of the vitamin E-binding protein afamin are associated with overall and progression-free survival and platinum sensitivity in serous ovarian cancer-a study by the OVCAD consortium. Gynecol. Oncol. 128(1), 38-43 (2013).

17. Penno MA, Klingler-Hoffmann M, Brazzatti JA et al. 2D-DIGE analysis of sera from transgenic mouse models reveals novel candidate protein biomarkers for human gastric cancer. J. Proteomics 77, 40-58 (2012).

18. Humphries JM, Penno MA, Weiland F et al. Identification and validation of novel candidate protein biomarkers for the detection of human gastric cancer. Biochim. Biophys. Acta 1844(5), 1051-1058 (2014).

19. Tolek A, Wongkham C, Proungvitaya $S$ et al. Serum alpha1beta-glycoprotein and afamin ratio as potential diagnostic and prognostic markers in cholangiocarcinoma. Exp. Biol. Med. (Maywood) 237(10), 1142-1149 (2012).

20. Choi JW, Liu H, Shin DH et al. Proteomic and cytokine plasma biomarkers for predicting progression from colorectal adenoma to carcinoma in human patients. Proteomics 13(15), 2361-2374 (2013).

21. Jeong DH, Kim HK, Prince AE et al. Plasma proteomic analysis of patients with squamous cell carcinoma of the uterine cervix. J. Gynecol. Oncol. 19(3), 173-180 (2008).

22. Opstal-Van Winden AW, Krop EJ, Karedal MH et al. Searching for early breast cancer biomarkers by serum protein profiling of pre-diagnostic serum; a nested case-control study. BMC Cancer 11, 381 (2011).

23. Song HJ, Xue YL, Qiu ZL, Luo QY. Comparative serum proteomic analysis identified afamin as a downregulated protein in papillary thyroid carcinoma patients with non-131I-avid lung metastases. Nucl. Med. Commun. 34(12), 1196-1203 (2013).

24. Shen CT, Wei WJ, Qiu ZL, Song HJ, Luo QY. Afamin promotes glucose metabolism in papillary thyroid carcinoma. Mol. Cell. Endocrinol. 434, 108-115 (2016).

25. Wu GX, Lin YM, Zhou TH, Gao H, Pei G. Significant down-regulation of alpha-albumin in human hepatoma and its implication. Cancer Lett. 160(2), 229-236 (2000).

26. Pang L, Li QQ, Li Y, Liu Y, Duan N, Li HX. Urine proteomics of primary membranous nephropathy using nanoscale liquid chromatography tandem mass spectrometry analysis. Clin. Proteomics 15, 5 (2018).

- Discovered uAFM as a biomarker candidate for primary membranous nephropathy by nanoscale LC-MS/MS analysis.

27. Rao PV, Lu X, Standley M et al. Proteomic identification of urinary biomarkers of diabetic nephropathy. Diabetes Care 30(3), 629-637 (2007).

28. Mucha K, Bakun M, Jazwiec R et al. Complement components, proteolysis-related, and cell communication-related proteins detected in urine proteomics are associated with IgA nephropathy. Pol. Arch. Med. Wewn. 124(7-8), 380-386 (2014).

29. Levey AS, Stevens LA, Schmid CH et al. A new equation to estimate glomerular filtration rate. Ann. Intern. Med. 150(9), 604-612 (2009).

30. Clinical and Laboratory Standards Institute (CLSI). User verification of performance for precision and trueness; approved guideline. In: CLSI Document EP15-A2 (2nd Edition). Clinical and laboratory standards Institute, PA, USA (2005).

31. Clinical and Laboratory Standards Institute (CLSI). Evaluation of the linearity of quantitative measurement procedures: a statistical approach; approved guideline. In: CLSI Document EP6-A. Clinical and laboratory standards Institute PA, USA (2003).

32. Clinical and Laboratory Standards Institute (CLSI). Protocols for determination of limits of detection and limits of quantitation; approved guideline. In: CLSI Document EP17-A. Clinical and laboratory standards Institute PA, USA (2004).

33. Clinical and Laboratory Standards Institute (CLSI). Defining, establishing, and verifying reference intervals in the clinical laboratory; approved Guideline. In: CLSI Document C28-A3 (3rd Edition). Clinical and laboratory standards Institute PA, USA (2008).

34. Shao C, Wang Y, Gao Y. Applications of urinary proteomics in biomarker discovery. Sci. China Life Sci. 54(5), 409-417 (2011).

35. Dieplinger H, Dieplinger B. Afamin-A pleiotropic glycoprotein involved in various disease states. Clin. Chim. Acta 446, 105-110 (2015).

36. Vivekanandan-Giri A, Slocum JL, Buller CL et al. Urine glycoprotein profile reveals novel markers for chronic kidney disease. Int. J. Proteomics 2011, 214715 (2011).

37. Geyer PE, Holdt LM, Teupser D, Mann M. Revisiting biomarker discovery by plasma proteomics. Mol. Syst. Biol. 13(9), 942 (2017).

38. Topbas C, Swick A, Razavi M, Anderson NL, Pearson TW, Bystrom C. Measurement of lipoprotein-associated phospholipase A2 by use of 3 different methods: exploration of discordance between ELISA and activity assays. Clin. Chem. 64(4), 697-704 (2018).

39. Heiser M, Hutter-Paier B, Jerkovic L et al. Vitamin E binding protein afamin protects neuronal cells in vitro. J. Neural Transm. Suppl. (62), 337-345 (2002).

40. Jing W, Vaziri ND, Nunes A et al. LCZ696 (Sacubitril/valsartan) ameliorates oxidative stress, inflammation, fibrosis and improves renal function beyond angiotensin receptor blockade in CKD. Am. J. Transl. Res. 9(12), 5473-5484 (2017).

41. Volpe CMO, Villar-Delfino PH, Dos Anjos PMF, Nogueira-Machado JA. Cellular death, reactive oxygen species (ROS) and diabetic complications. Cell Death Dis. 9(2), 119 (2018). 
